\title{
Major dietary patterns in relation to demographic and socio-economic status and food insecurity in two Iranian ethnic groups living in Urmia, Iran
}

\author{
Arezoo Rezazadeh 1,2, Nasrin Omidvar 1,*, Hassan Eini-Zinab ${ }^{1}$, Mahmoud Ghazi-Tabatabaie ${ }^{3}$, \\ Reza Majdzadeh ${ }^{4}$, Saeid Ghavamzadeh ${ }^{5}$ and Sakineh Nouri-Saeidlou ${ }^{6}$ \\ 'Department of Community Nutrition, National Nutrition and Food Technology Research Institute, Faculty of Nutrition \\ and Food Technology, Shahid Beheshti University of Medical Sciences and Health Services, No. 46 Hafezi Street, \\ Farahzadi Boulevard, Shahrak Ghods, PO Box 1981619573, Tehran, Islamic Republic of Iran: 'Students' Research \\ Committee, Shahid Beheshti University of Medical Sciences and Health Services, Tehran, Islamic Republic of Iran: \\ ${ }^{3}$ Department of Demography, Faculty of Social Science, Tehran University, Tehran, Islamic Republic of Iran: \\ ${ }^{4}$ Knowledge Utilization Research Center, Tehran University of Medical Sciences, Tehran, Islamic Republic of Iran: \\ ${ }^{5}$ Nutrition Sciences Department, Faculty of Medicine, Urmia University of Medical Sciences, Urmia, Islamic Republic of Iran: \\ ${ }^{6}$ Food and Beverages Safety Research Center, Urmia University of Medical Sciences, Urmia, Islamic Republic of Iran
}

Submitted 15 January 2015: Final revision received 16 April 2016: Accepted 20 May 2016: First published online 30 June 2016

\begin{abstract}
Objective: To identify major dietary patterns and their association with socioeconomic status (SES) and food insecurity in two major ethnic groups living in Urmia, north-west Iran.

Design: A cross-sectional study.

Setting: All four geographical zones of Urmia city.

Subjects: Participants ( $n$ 723; 427 women and 296 men), aged 20-64 years, from two ethnic groups (445 Azeri Turks and 278 Kurds).

Results: Three major dietary patterns were extracted: 'Traditional High SES' (THS), 'Traditional Low SES' (TLS) and 'Transitional'. After adjusting for confounders, the THS pattern was positively associated with education level and negatively associated with moderate or severe food insecurity in Azeri Turks; whereas, among Kurds, it was more common in women and positively associated with age. The TLS pattern was more common among men and negatively associated with educational level and all levels of food insecurity in Azeris; while, among Kurds, it was more common among men, positively associated with being married and negatively associated with household income/capita. The 'Transitional' pattern was positively associated with being employed and negatively associated with age and all levels of food insecurity in Azeris; while, among Kurds, it was more common among men and negatively associated with age, being married and physical activity level.

Conclusions: Findings suggest that household SES and food insecurity are associated with detrimental dietary patterns and that this effect may be stronger than cultural and ethnic background. These patterns differ by age and gender. Therefore, such characteristics should be considered in planning and formulating diet-related policies and programmes.
\end{abstract}

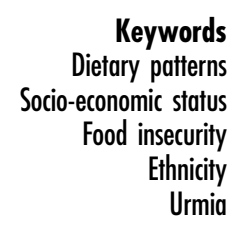

The role of diet in the development of chronic diseases is well documented ${ }^{(1,2)}$. Formulating appropriate food-based dietary recommendations for the general public requires a better understanding of dietary habits and their diversity ${ }^{(3)}$. Dietary pattern analysis defined by principal components is a complementary approach to more traditional analyses. Such an analysis represents a complex set of highly correlated dietary components in a factor (dietary pattern) that is representative of the characteristics of all its constituent food groups and their synergistic or antagonistic effects, rather than focusing on a single nutrient or food group in relation to health outcomes ${ }^{(4,5)}$. Dietary patterns are likely to vary according to gender, socioeconomic status (SES) and ethnicity ${ }^{(6,7)}$. As Gilbert and Khokhar $^{(8)}$ pointed out, in ethnic populations, dietary habits are affected by various factors, including food availability, food beliefs, health status, dietary laws and religious beliefs, cultural norms, income level and 
occupational status, as well as demographic factors such as birthplace and age. In a given environment with defined food availability, ethnicity and SES are two major determinants of dietary pattern ${ }^{(9)}$. Studies have suggested an attenuation of geographical differences in dietary behaviours $^{(10)}$ and the strong contribution of socioeconomic factors ${ }^{(11,12)}$.

Iran is a multi-ethnic country experiencing an accelerated nutrition and health transition ${ }^{(13)}$. Meanwhile, the present knowledge of various features of Iranian ethnic groups in terms of their health status and dietary habits is limited. To our knowledge, no studies have assessed the dietary habits of various Iranian ethnicities and factors contributing to the food habits of each ethnicity.

West Azerbaijan Province, located in the north-west of Iran and close to Turkey and Iraqi Kurdistan, includes a heterogeneous population in terms of ethnicities (including Azeris (76.2\%), Kurds (21.7\%), Persians (0.8\%) and others (1.4\%)) and religions (Shiite, Sunni, Christian and Assyrian) ${ }^{(14)}$. Urmia, as the capital city of the province, has a diverse ethnic composition too ${ }^{(15)}$; from which Azeri Turks and Kurds are the major ethnic groups. Urmia city has not been recognized as a metropolitan city yet and has not been much affected by acculturation; therefore, it provides a relatively appropriate environment in which to study the actual dietary behaviours and lifestyles and their relationships with SES of the resident ethnic groups. Therefore, the present study aimed to investigate the major dietary patterns and their possible association with SES, food insecurity and lifestyle factors in two Iranian ethnic groups living in Urmia.

\section{Methods}

\section{Participants}

The current cross-sectional study was part of a larger project entitled 'Designing and testing of a multilevel model to explain the effects of neighbourhood, household and individual levels on anthropometric factors in men and women living in the city of Urmia' funded by the National Nutrition and Food Technology Research Institute. The sample size of the main study was 723 (445 (61.5\%) Azeri Turks and 278 (38.5\%) Kurds).*

* The sample size calculation in the main study was based on BMI as the main dependent variable. According to the last report of the national project of 'Non-communicable Diseases Risk Factors' in $2008^{(16)}$, mean (95\% CI) BMI in 500 women and 500 men living in West Azerbaijan was $26.6(27.1,26.1)$ and $24.6(25.2,24.0) \mathrm{kg} / \mathrm{m}^{2}$, respectively. Standard deviation (SD) was calculated using the formulas: error $=Z(\mathrm{SE})$ and $\mathrm{SE}=\mathrm{SD} / \sqrt{ } N$, and considering the statistical power equal to $80 \%$, the $Z \mathrm{CI}$ (95\%) equal to 1.96 and $d=0.6 \mathrm{~kg} / \mathrm{m}^{2}$, the sample size was calculated using the following formula (where $\alpha=$ Type I error and $S=$ variance):

$$
n=\frac{S^{2} \times Z_{1-\alpha / 2}^{2}}{d^{2}} .
$$

Calculated sample size was 322 women and 462 men aged 20-64 years and the largest sample size (462) was selected. In order to increase the power of the study, 723 participants were recruited based on the gender and ethnic proportion in Urmia city, 445 (61.5\%) Azeri Turks and 278 (38.5\%) Kurds.
In order to conduct the current research to explore major dietary patterns and their socio-economic correlates, a power analysis based on previous research on Iranians ${ }^{(17)}$ approved that the aforementioned sample size was sufficient to detect similar results (power 90\%). Samples were selected by a combination of cluster, random and systematic sampling methods. Samples were selected from all four geographical zones (north, south, east and west) of Urmia. Health centres (clusters) were randomly selected based on population size at each zone. At each centre, a systematic random sampling approach was used to select households from the routine data registry, weighting by the population size at each centre. In each centre, the first household of each cluster was selected from routine data registry of the centre. Then the rest of the households in the cluster of ten were selected from both sides of the first household's home address (five households from the right and four from the left side of that house), despite being registered or not in the health centres. Two members of the household (one man and one woman) were interviewed by trained local nutritionists who were fluent in Turkish and Kurdish. Women interviewees substituted uncooperative male respondents for some households. The response rate was approximately $80 \%$ within the cases. The inclusion criteria were current residency in Urmia, belonging to either Azeri or Kurd ethnic group, not currently pregnant or lactating, and no history of endocrine or metabolic disorders. Individuals who had left $>50 \%$ of food items on the FFQ blank or those who misreported energy intake based on the Goldberg et $a l^{(18)}$ method were excluded from the study.

\section{Pilot study}

A pilot study was carried out on thirty individuals who were not subsequently included in the main study. The aim of the pilot study was to minimize errors and biases in data collection. During this step, the interviewers were trained and their interview skills were improved. Additionally, necessary modifications in the questionnaire were applied. Furthermore, viability of the study and cooperation of the participants were assessed.

\section{Assessment of dietary intake}

Data on dietary intake over a year were collected with a semi-quantitative 168-item FFQ. The FFQ was a Willettformat questionnaire modified based on Iranian food items and it was used to obtain information on dietary intake over a 1-year period ${ }^{(19)}$. It included a list of foods (with standard serving sizes) commonly consumed by Iranians. Individuals were requested to report their frequency of consumption of a given serving of each food item, during the past year, on a daily, weekly, monthly or yearly basis. Validity and reproducibility of this FFQ have been reported ${ }^{(20,21)}$ and approved for the Iranian population ${ }^{(22)}$. However, taking into account that our studied population included two 
Iranian ethnic groups, content validity of the FFQ was also assessed by five local nutrition experts in the nutrition department of Urmia University of Medical Sciences. Based on their recommendations, eight commonly consumed food items including ash (a thick soup), dolma (stuffed grape, cabbage or other vegetable leaves), kofte (meatballs), khagineh (traditional pancake), walnut \& carrot halva (traditional sweet), herbal beverages (pussy willow and lemon balm) and turkey were added to the FFQ food list. After converting the reported frequency of each food item to its daily intake value, portion sizes of consumed foods were converted to grams using household measures $^{(23)}$. In the next step, food items were categorized into thirty-three predefined food groups. Food grouping was performed based on previous studies ${ }^{(22,24,25)}$. In addition, some food items that were indicative of distinct dietary patterns in the studied population such as traditional foods (e.g. kofte, dolma, khagineh) were grouped separately.

\section{Assessment of demographic and socio-economic characteristics}

Demographic (age, gender, duration of residence in Urmia, marital status, household size, number of children) and socio-economic (educational status, occupation, total income/capita, total expenses/capita, household economic status) characteristics were collected by questionnaire. Occupation was categorized into five levels: (i) unemployed and/or housewife; (ii) job category 1 (labourer, farmer, rancher); (iii) job category 2 (self-employed, shopkeeper); (iv) job category 3 (teacher, serviceman, government employee, trooper); and (v) job category 4 (employer, industrialist, business owner, manager, doctor, lawyer, pilot, university professor). Household economic status was defined by scoring household assets based on their economic value according to a previous study ${ }^{(26)}$ and computing the total score. The total score was categorized into tertiles (T) and T1, T2 and T3 were labelled as low, middle and high SES, respectively.

\section{Food security status}

Household food security was measured using a locally adapted Household Food Insecurity Access Scale (HFIAS) $^{(27)}$. The scale consisted of nine items with four frequency options that classified households into secure, mildly insecure, moderately insecure and severely insecure (food insecurity) categories. Validity of the HFIAS in the Iranian population has been confirmed previously ${ }^{(28)}$. All data were collected through face-to-face interviews with heads of households or their spouses at their home.

\section{Statistical methods}

Data were analysed using the statistical software package SPSS Version 16.0. Principal components analysis was used to identify major dietary patterns based on the thirty-three predefined food groups. Factors with eigenvalues $\geq 1.0$ were retained. Selection of the factors was based on the inflection of the scree test and interpretability. Varimax rotation was applied as a solution to obtain an orthogonal rotation to simplify the factor structure and render it more easily interpretable. The derived factors were labelled on the basis of interpretation of the data and the earlier literature. The factor score for each pattern was calculated by summing intakes of food groups weighted by the factor loading ${ }^{(29)}$ and each individual received a factor score for each identified pattern.

To compare general characteristics of the two ethnic groups across tertiles of identified dietary patterns, one-way ANOVA and $\chi^{2}$ tests were used wherever applicable. In both ethnic groups, the relationship between demographic, socio-economic and lifestyle variables and adherence to each dietary pattern was assessed by ANCOVA. Data were simultaneously adjusted for all variables (demographic, socio-economic, lifestyle), energy intake $(\mathrm{kJ} / \mathrm{d})$ and BMI. $P<0.05$ was considered as significant.

\section{Results}

Data were analysed for 695 participants. Twenty (3.8\%) individuals (twelve Azeri Turks and eight Kurds) were excluded due to misreporting of energy intake and eight $(1 \cdot 1 \%)$ individuals (three Azeri Turks and five Kurds) were excluded due to incomplete FFQ ( $>50 \%$ of items blank).

Three major dietary patterns were extracted through factor analysis: (i) 'Traditional High SES' (THS), with high loadings for fruit and natural juice, cabbage, dark green leafy vegetables, dairy products, dried and canned fruits, other vegetables, olives and nuts, traditional herbal infusions, khagineh, dolma, ash and pickles; (ii) 'Transitional', with high loadings for fast foods, red meat and organ meat, kebab, salty snacks, sauces (ketchup and mayonnaise), sweetened drinks, sweets and confectionery, poultry, fish and shrimp, seeds and coffee; and (iii) 'Traditional Low SES' (TLS), with high loadings for tea, refined grains, potatoes, whole grains, hydrogenated oils and animal fat, abgoosht (a traditional stew), salt, vegetable oil, eggs and legumes (Table 1).

Taken together, a higher percentage of Azeri Turks (40.5\%) and a lower percentage of Kurds (23.6\%) were in the highest intake tertile of the THS dietary pattern $(P=0.00)$. In contrast, a higher percentage of Kurds $(37.9 \%)$ were at the highest intake tertile, while $36.0 \%$ of Azaris were in the lowest intake tertile of the TLS dietary pattern $(P=0.01)$. The distributions of the two ethnic groups across tertiles of the 'Transitional' dietary pattern were not significantly different.

\section{The 'Traditional High Socio-economic Status' dietary pattern}

As shown in Table 2, among Azeri participants, a greater percentage of those in the highest tertile of the THS dietary 
Table 1 Factor loading matrix for the major dietary patterns identified using FFQ data among two Iranian ethnic groups living in Urmia, Iran

\begin{tabular}{|c|c|c|c|}
\hline \multirow[b]{2}{*}{ Food group } & \multicolumn{3}{|c|}{ Major dietary pattern } \\
\hline & $\begin{array}{l}\text { 'Traditional } \\
\text { Higher } \\
\text { SES' }\end{array}$ & 'Transitional' & $\begin{array}{l}\text { 'Traditional } \\
\text { Lower } \\
\text { SES' }\end{array}$ \\
\hline Fruits and natural juice & 0.673 & & \\
\hline Cabbages & 0.589 & & \\
\hline $\begin{array}{l}\text { Dark green leafy } \\
\text { vegetables }\end{array}$ & 0.560 & & \\
\hline Dairy products & 0.543 & & \\
\hline Canned and dried fruits & 0.493 & 0.207 & \\
\hline Other vegetables & 0.461 & & 0.357 \\
\hline Olives and nuts & 0.457 & 0.218 & \\
\hline $\begin{array}{l}\text { Herbal beverages (pussy } \\
\text { willow and lemon balm) }\end{array}$ & 0.373 & & \\
\hline $\begin{array}{l}\text { Khagineh (traditional } \\
\text { pancake) }\end{array}$ & 0.272 & & \\
\hline $\begin{array}{l}\text { Dolma (stuffed grape, } \\
\text { cabbage or other } \\
\text { vegetable leaves) }\end{array}$ & 0.259 & & \\
\hline Ash (traditional thick soup) & 0.251 & & \\
\hline Pickles & 0.249 & & \\
\hline Fast foods & & 0.673 & \\
\hline Red meat and organ meat & & 0.666 & \\
\hline Kebab & & 0.566 & \\
\hline Salty snacks & & 0.512 & \\
\hline $\begin{array}{l}\text { Dressings (ketchup and } \\
\text { mayonnaise) }\end{array}$ & & 0.489 & \\
\hline Sweets and sweet drinks & & 0.484 & \\
\hline Poultry and fish & & 0.397 & 0.213 \\
\hline Seeds & & 0.275 & \\
\hline Coffee & & 0.257 & \\
\hline \multicolumn{4}{|l|}{ Kofte (meatballs) } \\
\hline Stew & & & \\
\hline Tea & & & 0.534 \\
\hline Refined grains & & 0.224 & 0.512 \\
\hline Potato & & & 0.512 \\
\hline Whole grains & & & 0.505 \\
\hline $\begin{array}{l}\text { Hydrogenated and animal } \\
\text { fats }\end{array}$ & & & 0.428 \\
\hline Broth & & & 0.423 \\
\hline Salt & & & 0.414 \\
\hline Liquid oil & & & 0.359 \\
\hline Egg & & 0.210 & 0.277 \\
\hline Legumes & & & 0.267 \\
\hline
\end{tabular}

SES, socio-economic status.

Values $<0.20$ were excluded for simplicity.

Total variance explained by three factors: $22.6 \%$ ('Traditional Higher SES',

'Transitional' and 'Traditional Lower SES' explained 10.3, 6.3 and $6.0 \%$ of

the total variance, respectively).

pattern were women, non-smokers, from middle- and high-SES households who were either food secure or with mild food insecurity. Those in the middle tertile of the THS dietary pattern had higher per capita income and expenses. Among Kurds, those categorized in the highest tertile of the THS dietary pattern had higher per capita income and expenses than those in lower tertiles.

\section{The 'Transitional' dietary pattern}

Azeri individuals in the highest tertile of the 'Transitional' dietary pattern were younger, not married, had a lower duration of residence in Urmia and fewer children than those in lower tertiles. Further, more in this tertile, $v$. lower tertiles, had 6-12 years of education or a university degree and lived in food-secure households. Among Kurds, individuals in the highest tertile of the 'Transitional' dietary pattern tended to be younger, not married and with fewer children, higher income and expenses per capita, in foodsecure households and with 6-12 years of education or a university degree (Table 3).

\section{The 'Traditional Low Socio-economic Status' dietary pattern}

Among Azeri Turks, more men and those working in job categories 1 and 2 were categorized in the highest $v$. the lower tertiles of the TLS dietary pattern. Kurds in the highest tertile of the TLS dietary pattern were also more likely to be employed in the job categories 1 and 2, with lower per capita income and expenses relative to those in lower tertiles of the TLS dietary pattern (Table 4).

\section{Factors associated with adberence to the three dietary patterns}

After adjusting for confounders and all variables in the model, the THS dietary pattern was positively associated with age, intermediate and university degrees (relative to illiteracy and primary education) and negatively associated with moderate or severe food insecurity (relative to food secure). Among Kurds, the THS dietary pattern was positively associated with age and was more common among women than men (Table 5). On the other hand, the 'Transitional' dietary pattern was positively associated with employment (relative to being a housewife or unemployed) and negatively associated with age and mild, moderate or severe food insecurity ( $v$. food secure) among Azeri Turks. Among Kurds, the THS dietary pattern was more common among men and negatively associated with age, being married, and mild, moderate or severe food insecurity. Finally, the TLS dietary pattern was more common among men, positively associated with mild, moderate or severe food insecurity, and negatively associated with intermediate or university education in Azeri Turks. The TLS dietary pattern was positively associated with being married and negatively associated with household income per capita in Kurds.

\section{Discussion}

To our knowledge, the present study is the first conducted among Iranian ethnic groups to define their major dietary patterns and to discover their probable association with SES and food insecurity. Most previous studies on Iranian dietary patterns were done with residents of the capital city, Tehran, without considering ethnicity. In the present study, three major dietary patterns were identified in a representative sample of Azeri and Kurd ethnic groups 
Table 2 Major characteristics of study participants according to tertiles of the 'Traditional Higher SES' dietary pattern and ethnic group, Urmia, Iran

\begin{tabular}{|c|c|c|c|c|c|c|c|c|c|c|c|c|c|c|}
\hline & \multicolumn{7}{|c|}{ Azeri Turks } & \multicolumn{7}{|c|}{ Kurds } \\
\hline & \multicolumn{2}{|c|}{$\mathrm{T} 1$} & \multicolumn{2}{|c|}{$\mathrm{T} 2$} & \multicolumn{2}{|c|}{ T3 } & \multirow[b]{2}{*}{$P \dagger$} & \multicolumn{2}{|c|}{$\mathrm{T} 1$} & \multicolumn{2}{|c|}{$\mathrm{T} 2$} & \multicolumn{2}{|c|}{ T3 } & \multirow[b]{2}{*}{$P \dagger$} \\
\hline & $n$ & $\%$ & $n$ & $\%$ & $n$ & $\%$ & & $n$ & $\%$ & $n$ & $\%$ & $n$ & $\%$ & \\
\hline \multicolumn{15}{|l|}{ Gender } \\
\hline Women & 47 & $20 \cdot 3$ & 81 & $35 \cdot 1$ & 103 & 44.6 & \multirow[t]{2}{*}{0.00} & 65 & 43.6 & 50 & 33.6 & 34 & $22 \cdot 8$ & \multirow[t]{2}{*}{0.81} \\
\hline Men & 56 & 34.6 & 50 & 30.9 & 56 & 34.6 & & 44 & 39.6 & 40 & $36 \cdot 0$ & 27 & $24 \cdot 3$ & \\
\hline \multicolumn{15}{|l|}{ Marital status } \\
\hline Married & 78 & $26 \cdot 1$ & 96 & $32 \cdot 1$ & 125 & 41.8 & \multirow[t]{2}{*}{0.56} & 73 & $40 \cdot 8$ & 57 & 31.8 & 49 & 27.4 & \multirow[t]{2}{*}{0.07} \\
\hline Single/divorced/widow & 25 & $26 \cdot 6$ & 35 & $37 \cdot 2$ & 34 & 21.4 & & 36 & $44 \cdot 4$ & 33 & 40.7 & 12 & 14.8 & \\
\hline \multicolumn{15}{|l|}{ Educational level } \\
\hline Illiterate and $\leq 5$ years & 45 & $32 \cdot 6$ & 41 & 29.7 & 52 & $37 \cdot 7$ & \multirow[t]{3}{*}{0.21} & 82 & 43.2 & 65 & $34 \cdot 2$ & 43 & $22 \cdot 6$ & \multirow[t]{3}{*}{0.49} \\
\hline $6-12$ years & 27 & $20 \cdot 1$ & 47 & $35 \cdot 1$ & 60 & $44 \cdot 8$ & & 17 & 40.5 & 12 & 28.6 & 13 & 31.0 & \\
\hline University degree & 31 & $25 \cdot 6$ & 43 & 35.5 & 47 & 38.8 & & 10 & 35.7 & 13 & 46.4 & 5 & 17.9 & \\
\hline \multicolumn{15}{|l|}{ Job status $\ddagger$} \\
\hline Unemployed, housewife & 50 & $27 \cdot 4$ & 78 & $35 \cdot 0$ & 95 & $42 \cdot 6$ & \multirow[t]{4}{*}{0.37} & 66 & $45 \cdot 3$ & 57 & $35 \cdot 8$ & 36 & $22 \cdot 6$ & \multirow[t]{3}{*}{0.44} \\
\hline Job category 1 and 2 & 21 & 33.9 & 18 & 29.0 & 23 & $31 \cdot 1$ & & 39 & $45 \cdot 3$ & 25 & $29 \cdot 1$ & 22 & $25 \cdot 6$ & \\
\hline Job category 3 and 4 & 32 & $29 \cdot 6$ & 35 & $32 \cdot 4$ & 41 & 38.0 & & 4 & $26 \cdot 7$ & 8 & 53.3 & 3 & $20 \cdot 0$ & \\
\hline \multicolumn{14}{|l|}{ SES } & \\
\hline Low & 38 & $30 \cdot 6$ & 48 & 38.7 & 38 & 30.6 & \multirow[t]{3}{*}{0.03} & 44 & $45 \cdot 4$ & 33 & 34.0 & 20 & $20 \cdot 6$ & \multirow[t]{3}{*}{0.48} \\
\hline Middle & 29 & $24 \cdot 0$ & 44 & $36 \cdot 4$ & 48 & $39 \cdot 7$ & & 33 & 35.4 & 37 & 39.8 & 23 & 24.7 & \\
\hline High & 36 & 24.5 & 39 & $26 \cdot 5$ & 72 & $49 \cdot 0$ & & 32 & $45 \cdot 7$ & 20 & 28.6 & 18 & $25 \cdot 7$ & \\
\hline \multicolumn{15}{|l|}{ Food insecurity status } \\
\hline Secure & 48 & $21 \cdot 0$ & 74 & $32 \cdot 3$ & 107 & $46 \cdot 7$ & 0.01 & 49 & 38.6 & 40 & 31.5 & 38 & 29.9 & 0.24 \\
\hline Mild insecure & 31 & $32 \cdot 0$ & 32 & 33.0 & 34 & $35 \cdot 1$ & & 22 & $39 \cdot 3$ & 25 & 44.6 & 9 & $16 \cdot 1$ & \\
\hline Moderate and severe & 23 & $37 \cdot 1$ & 23 & $37 \cdot 1$ & 16 & $25 \cdot 8$ & & 37 & $48 \cdot 7$ & 25 & $32 \cdot 9$ & 14 & $18 \cdot 4$ & \\
\hline & Mean & SD & Mean & SD & Mean & SD & $P †$ & Mean & SD & Mean & SD & Mean & SD & $P+$ \\
\hline Age (years) & 39.6 & $13 \cdot 1$ & $39 \cdot 0$ & $12 \cdot 9$ & 41.8 & $12 \cdot 4$ & 0.15 & $32 \cdot 3$ & $11 \cdot 2$ & 34.5 & $12 \cdot 4$ & 34.8 & $10 \cdot 9$ & 0.29 \\
\hline Household size (no. of persons) & 3.8 & 1.0 & 4.0 & $1 \cdot 1$ & 3.8 & 1.0 & 0.77 & $6 \cdot 2$ & $2 \cdot 2$ & $5 \cdot 9$ & $2 \cdot 0$ & 5.5 & 1.6 & 0.08 \\
\hline Number of children & 1.9 & 1.5 & 2.0 & 1.1 & $2 \cdot 1$ & 1.2 & 0.56 & 2.9 & 2.5 & 3.0 & 2.6 & $2 \cdot 7$ & $2 \cdot 2$ & 0.68 \\
\hline Duration of residence (years) & 28.6 & $16 \cdot 8$ & $31 \cdot 2$ & $16 \cdot 0$ & 32.7 & $17 \cdot 0$ & 0.14 & 20.7 & 11.0 & $24 \cdot 3$ & 12.1 & 23.0 & 11.1 & 0.08 \\
\hline Total income/capita (10 000 Rials) & $325 \cdot 3$ & 269.5 & $465 \cdot \overline{5}$ & $610 \cdot 6$ & 423.5 & 278.0 & 0.03 & 240.7 & $173 \cdot 3$ & 238.9 & 189.2 & 352.0 & 235.2 & 0.001 \\
\hline Total expenses/capita (10 000 Rials) & $276 \cdot 3$ & $215 \cdot 0$ & 429.2 & 592.0 & 332.7 & $197 \cdot 1$ & 0.00 & 211.8 & 120.7 & 253.7 & 135.5 & $290 \cdot 3$ & 184.2 & 0.002 \\
\hline
\end{tabular}

SES, socio-economic status; $\mathrm{T}$, tertile ( $\mathrm{T} 1=$ low, $\mathrm{T} 2=$ middle; $\mathrm{T} 3=$ high).

appropriate.

$\ddagger$ Job category 1 = labourer, farmer, rancher; job category 2 =self-employed, shopkeeper; job category $3=$ teacher, serviceman, government employee, trooper; job category $4=$ employer, industrialist, business owner, manager, doctor, lawyer, pilot, university professor. 
Table 3 Major characteristics of study participants according to tertiles of the 'Transitional' dietary pattern and ethnic group, Urmia, Iran

\begin{tabular}{|c|c|c|c|c|c|c|c|c|c|c|c|c|c|c|}
\hline & \multicolumn{7}{|c|}{ Azeri Turks } & \multicolumn{7}{|c|}{ Kurds } \\
\hline & \multicolumn{2}{|c|}{$\mathrm{T} 1$} & \multicolumn{2}{|c|}{ T2 } & \multicolumn{2}{|c|}{ T3 } & \multirow[b]{2}{*}{$P \dagger$} & \multicolumn{2}{|c|}{$\mathrm{T} 1$} & \multicolumn{2}{|c|}{ T2 } & \multicolumn{2}{|c|}{ T3 } & \multirow[b]{2}{*}{$P \dagger$} \\
\hline & $n$ & $\%$ & $n$ & $\%$ & $n$ & $\%$ & & $n$ & $\%$ & $n$ & $\%$ & $n$ & $\%$ & \\
\hline \multicolumn{15}{|l|}{ Gender } \\
\hline Women & 74 & 31.6 & 89 & 38.0 & 71 & $30 \cdot 3$ & \multirow[t]{2}{*}{0.36} & 60 & $40 \cdot 8$ & 41 & 27.9 & 46 & 31.3 & \multirow[t]{2}{*}{0.16} \\
\hline Men & 41 & $26 \cdot 3$ & 58 & $37 \cdot 2$ & 57 & $36 \cdot 5$ & & 33 & 29.5 & 38 & 33.9 & 41 & $36 \cdot 6$ & \\
\hline \multicolumn{15}{|l|}{ Marital status } \\
\hline Married & 100 & 33.2 & 114 & 37.9 & 87 & 28.9 & \multirow[t]{2}{*}{0.00} & 78 & $43 \cdot 3$ & 52 & 28.9 & 50 & $27 \cdot 8$ & \multirow[t]{2}{*}{0.00} \\
\hline Single/divorced/widow & 15 & $16 \cdot 9$ & 33 & 37.1 & 41 & $46 \cdot 1$ & & 15 & $19 \cdot 0$ & 27 & 34.2 & 37 & $46 \cdot 8$ & \\
\hline \multicolumn{15}{|l|}{ Educational level } \\
\hline Illiterate and $\leq 5$ years & 56 & $40 \cdot 6$ & 49 & $35 \cdot 5$ & 33 & 23.9 & \multirow[t]{3}{*}{0.00} & 82 & 43.9 & 55 & $29 \cdot 4$ & 50 & $26 \cdot 7$ & \multirow[t]{3}{*}{0.00} \\
\hline $6-12$ years & 38 & $28 \cdot 8$ & 48 & $36 \cdot 4$ & 46 & 34.8 & & 8 & $18 \cdot 6$ & 12 & 27.9 & 23 & 53.5 & \\
\hline University degree & 21 & 17.5 & 50 & 41.7 & 49 & $40 \cdot 8$ & & 3 & $10 \cdot 3$ & 12 & 41.4 & 14 & $48 \cdot 3$ & \\
\hline \multicolumn{15}{|l|}{ Job status $\ddagger$} \\
\hline Unemployed, housewife & 66 & $20 \cdot 7$ & 82 & $36 \cdot 9$ & 74 & $33 \cdot 3$ & \multirow[t]{4}{*}{0.62} & 61 & $39 \cdot 1$ & 44 & $28 \cdot 2$ & 51 & $32 \cdot 7$ & \multirow[t]{4}{*}{0.51} \\
\hline Job category 1 and 2 & 19 & $31 \cdot 1$ & 19 & $31 \cdot 1$ & 23 & 37.7 & & 28 & 32.6 & 30 & 34.9 & 28 & $32 \cdot 6$ & \\
\hline Job category 3 and 4 & 30 & $28 \cdot 0$ & 46 & 43.0 & 31 & $29 \cdot 0$ & & 4 & 23.5 & 5 & 29.4 & 8 & $47 \cdot 1$ & \\
\hline \multicolumn{13}{|l|}{ SES } & & \\
\hline Low & 35 & 28.5 & 54 & 43.9 & 34 & $27 \cdot 6$ & \multirow[t]{3}{*}{0.06} & 44 & $46 \cdot 3$ & 28 & 29.5 & 23 & $24 \cdot 2$ & \multirow[t]{3}{*}{0.06} \\
\hline Middle & 44 & 37.0 & 35 & 29.4 & 40 & 33.6 & & 29 & 31.2 & 28 & $30 \cdot 1$ & 36 & 38.7 & \\
\hline High & 36 & 24.5 & 57 & 38.8 & 54 & $36 \cdot 7$ & & 20 & 28.2 & 23 & 32.4 & 28 & $39 \cdot 4$ & \\
\hline \multicolumn{15}{|l|}{ Food insecurity status } \\
\hline Secure & 55 & $24 \cdot 2$ & 82 & $36 \cdot 1$ & 90 & 39.6 & 0.01 & 29 & 23.2 & 40 & $32 \cdot 0$ & 56 & $44 \cdot 8$ & 0.00 \\
\hline Mild insecure & 35 & $37 \cdot 2$ & 36 & 38.3 & 23 & 24.5 & & 26 & $45 \cdot 6$ & 16 & $28 \cdot 1$ & 15 & $26 \cdot 3$ & \\
\hline Moderate and severe & 23 & $35 \cdot 9$ & 27 & $42 \cdot 2$ & 14 & 21.9 & & 37 & $48 \cdot 7$ & 23 & $30 \cdot 3$ & 16 & $21 \cdot 1$ & \\
\hline & Mean & SD & Mean & SD & Mean & SD & $P+$ & Mean & SD & Mean & SD & Mean & SD & $P+$ \\
\hline Age (years) & $46 \cdot 8$ & $10 \cdot 5$ & $40 \cdot 6$ & $12 \cdot 5$ & $35 \cdot 1$ & 11.9 & 0.00 & $40 \cdot 2$ & 11.5 & $32 \cdot 0$ & 9.5 & $29 \cdot 0$ & 10.5 & 0.00 \\
\hline Household size (no. of persons) & 3.9 & 1.0 & 3.8 & 1.0 & 3.8 & $1 \cdot 1$ & 0.90 & $24 \cdot 8$ & $13 \cdot 1$ & $22 \cdot 4$ & $11 \cdot 2$ & $21 \cdot 0$ & $10 \cdot 5$ & 0.10 \\
\hline Number of children & $2 \cdot 3$ & $1 \cdot 1$ & $2 \cdot 1$ & 1.4 & 1.8 & 1.2 & 0.01 & $6 \cdot 1$ & 1.9 & 5.9 & $2 \cdot 2$ & 6.0 & $2 \cdot 1$ & 0.60 \\
\hline Duration of residence (years) & 35.8 & 17.3 & $31 \cdot 1$ & $17 \cdot 0$ & $27 \cdot 1$ & $15 \cdot 0$ & 0.00 & 4.0 & 2.4 & 2.5 & $2 \cdot 3$ & 2.0 & $2 \cdot 2$ & 0.00 \\
\hline Total income/capita (10000 Rials) & 369.7 & $382 \cdot 3$ & $422 \cdot 1$ & $500 \cdot 9$ & 456.9 & $354 \cdot 0$ & 0.27 & $226 \cdot 0$ & 171.6 & $262 \cdot 3$ & 204.0 & $317 \cdot 0$ & $227 \cdot 3$ & 0.01 \\
\hline Total expenses/capita (10000 Rials) & 330.6 & 359.8 & 377.5 & 484.2 & 342.8 & $260 \cdot 6$ & 0.58 & $222 \cdot 0$ & 152.1 & 239.7 & 134.3 & $277 \cdot 3$ & $162 \cdot 2$ & 0.04 \\
\hline
\end{tabular}

$\mathrm{SES}$, socio-economic status; $\mathrm{T}$, tertile $(\mathrm{T} 1=\mathrm{low}, \mathrm{T} 2=$ middle; $\mathrm{T} 3=$ high $)$

$\dagger P$ values were calculated by one-way ANOVA and the $x^{2}$ test where appropriate.

$\ddagger$ Job category $1=$ labourer, farmer, rancher; job category $2=$ self-employed, shopkeeper; job category $3=$ teacher, serviceman, government employee, trooper; job category $4=$ employer, industrialist, business owner,

manager, doctor, lawyer, pilot, university professor. 
Table 4 Major characteristics of study participants according to tertiles of the 'Traditional Lower SES' dietary pattern and ethnic group, Urmia, Iran

\begin{tabular}{|c|c|c|c|c|c|c|c|c|c|c|c|c|c|c|}
\hline & \multicolumn{7}{|c|}{ Azeri Turks } & \multicolumn{7}{|c|}{ Kurds } \\
\hline & \multicolumn{2}{|c|}{$\mathrm{T} 1$} & \multicolumn{2}{|c|}{$\mathrm{T} 2$} & \multicolumn{2}{|c|}{ T3 } & \multirow[b]{2}{*}{$P \dagger$} & \multicolumn{2}{|c|}{$\mathrm{T} 1$} & \multicolumn{2}{|c|}{$\mathrm{T} 2$} & \multicolumn{2}{|c|}{ T3 } & \multirow[b]{2}{*}{$P \dagger$} \\
\hline & $n$ & $\%$ & $n$ & $\%$ & $n$ & $\%$ & & $n$ & $\%$ & $n$ & $\%$ & $n$ & $\%$ & \\
\hline \multicolumn{15}{|l|}{ Gender } \\
\hline Women & 110 & $47 \cdot 0$ & 84 & 35.9 & 40 & $17 \cdot 1$ & \multirow[t]{2}{*}{0.00} & 50 & $33 \cdot 3$ & 54 & $36 \cdot 0$ & 46 & $30 \cdot 7$ & \multirow[t]{2}{*}{0.00} \\
\hline Men & 31 & $19 \cdot 6$ & 46 & $29 \cdot 1$ & 81 & 51.3 & & 16 & $14 \cdot 3$ & 42 & 37.5 & 54 & $48 \cdot 2$ & \\
\hline \multicolumn{15}{|l|}{ Marital status } \\
\hline Married & 100 & 33.4 & 101 & 33.8 & 98 & $32 \cdot 8$ & \multirow[t]{2}{*}{$0 \cdot 14$} & 42 & $23 \cdot 2$ & 68 & $37 \cdot 6$ & 71 & $39 \cdot 2$ & \multirow[t]{2}{*}{0.54} \\
\hline Single/divorced/widow & 41 & $44 \cdot 1$ & 29 & $31 \cdot 2$ & 23 & $24 \cdot 7$ & & 24 & $29 \cdot 6$ & 28 & 34.6 & 29 & $35 \cdot 8$ & \\
\hline \multicolumn{15}{|l|}{ Educational level } \\
\hline Illiterate \& and $\leq 5$ years & 41 & 29.9 & 42 & $30 \cdot 7$ & 54 & 39.4 & \multirow[t]{3}{*}{0.09} & 46 & $24 \cdot 2$ & 71 & 37.4 & 73 & 38.4 & \multirow[t]{3}{*}{0.75} \\
\hline $6-12$ years & 51 & $38 \cdot 3$ & 45 & 33.8 & 37 & $27 \cdot 8$ & & 10 & $23 \cdot 3$ & 17 & 39.5 & 16 & $37 \cdot 2$ & \\
\hline University degree & 49 & $40 \cdot 2$ & 43 & $35 \cdot 2$ & 30 & $24 \cdot 6$ & & 10 & 34.5 & 8 & $27 \cdot 6$ & 11 & $37 . \overline{9}$ & \\
\hline \multicolumn{15}{|l|}{ Job statusf } \\
\hline Unemployed, housewife & 91 & $40 \cdot 6$ & 77 & 34.4 & 56 & $25 \cdot 0$ & \multirow[t]{4}{*}{0.00} & 49 & $30 \cdot 8$ & 58 & 36.5 & 52 & $32 \cdot 7$ & \multirow[t]{4}{*}{0.00} \\
\hline Job category 1 and 2 & 12 & $19 \cdot 7$ & 16 & $26 \cdot 2$ & 33 & $54 \cdot 1$ & & 8 & $9 \cdot 3$ & 34 & 39.5 & 44 & $51 \cdot 2$ & \\
\hline Job category 3 and 4 & 38 & $35 \cdot 5$ & 37 & $34 \cdot 6$ & 32 & $29 \cdot 9$ & & 9 & 52.9 & 4 & 23.5 & 4 & 23.5 & \\
\hline \multicolumn{13}{|l|}{ SES } & & \\
\hline Low & 40 & $32 \cdot 3$ & 41 & $33 \cdot 1$ & 43 & $34 \cdot 7$ & \multirow[t]{3}{*}{0.81} & 18 & $18 \cdot 6$ & 36 & $37 \cdot 1$ & 43 & $44 \cdot 3$ & \multirow[t]{3}{*}{0.25} \\
\hline Middle & 45 & $37 \cdot 8$ & 39 & $32 \cdot 8$ & 35 & 29.4 & & 27 & $29 \cdot 0$ & 31 & $33 \cdot 3$ & 35 & $37 \cdot 6$ & \\
\hline High & 56 & $37 \cdot 8$ & 49 & $33 \cdot 1$ & 43 & $29 \cdot 1$ & & 21 & $29 \cdot 2$ & 29 & $40 \cdot 3$ & 22 & $36 \cdot 6$ & \\
\hline \multicolumn{15}{|l|}{ Food insecurity status } \\
\hline Secure & 92 & $40 \cdot 0$ & 75 & $32 \cdot 6$ & 63 & $27 \cdot 4$ & 0.07 & 41 & $31 \cdot 8$ & 49 & 38.0 & 39 & $30 \cdot 2$ & 0.03 \\
\hline Mild insecure & 33 & 34.4 & 34 & $35 \cdot 4$ & 29 & $30 \cdot 2$ & & 11 & $19 \cdot 6$ & 17 & $30 \cdot 4$ & 28 & $50 \cdot 0$ & \\
\hline Moderate and severe & 14 & $23 \cdot 0$ & 20 & $32 \cdot 8$ & 27 & $44 \cdot 3$ & & 13 & $17 \cdot 1$ & 30 & 39.5 & 33 & $43 \cdot 2$ & \\
\hline & Mean & SD & Mean & SD & Mean & SD & $P \dagger$ & Mean & SD & Mean & SD & Mean & SD & $P \dagger$ \\
\hline Age (years) & $39 \cdot 3$ & $12 \cdot 9$ & 39.9 & 12.5 & $42 \cdot 1$ & $12 \cdot 6$ & 0.18 & $32 \cdot 1$ & $10 \cdot 9$ & $34 \cdot 3$ & 11.9 & 34.4 & 11.9 & 0.39 \\
\hline Household size (no. of persons) & 3.8 & 1.04 & 3.9 & $1 \cdot 1$ & 3.8 & $1 \cdot 1$ & 0.61 & 5.5 & $2 \cdot 0$ & $6 \cdot 0$ & $2 \cdot 2$ & $6 \cdot 2$ & 1.9 & 0.11 \\
\hline Number of children & $2 \cdot 0$ & 1.03 & $2 \cdot 0$ & 1.5 & $2 \cdot 2$ & 1.2 & 0.24 & 2.3 & 2.0 & 3.1 & 2.6 & 3.1 & $2 \cdot 6$ & 0.12 \\
\hline Duration of residence (years) & 31.9 & $15 \cdot 3$ & $31 \cdot 2$ & $16 \cdot 9$ & 30.9 & $18 \cdot 0$ & 0.87 & $25 \cdot 0$ & $11 \cdot 8$ & 21.4 & $12 \cdot 3$ & $22 \cdot 6$ & $11 \cdot 0$ & 0.14 \\
\hline Total income/capita (10000 Rials) & $416 \cdot 7$ & 386.6 & $415 \cdot 3$ & $312 \cdot 9$ & $416 \cdot 8$ & $545 \cdot 0$ & 0.99 & 371.6 & 284.3 & $260 \cdot 9$ & $191 \cdot 2$ & 217.5 & $122 \cdot 0$ & 0.00 \\
\hline Total expenses/capita (10 000 Rials) & 369.4 & 354.0 & 324.9 & 274.5 & $356 \cdot 3$ & 534.0 & 0.62 & 293.8 & 182.6 & $240 \cdot 4$ & 168.9 & 228.6 & 104.7 & 0.02 \\
\hline
\end{tabular}

SES, socio-economic status; $\mathrm{T}$, tertile ( $\mathrm{T} 1=$ low, $\mathrm{T} 2=$ middle; $\mathrm{T} 3=$ high).

$P$ values were calculated by one-way ANOVA and the $x^{2}$ test where appropriate.

†Job category 1 = labourer, farmer, rancher; job category $2=$ self-employed, shopkeeper; job category $3=$ teacher, serviceman, government employee, trooper; job category $4=$ employer, industrialist, business owner, manager, doctor, lawyer, pilot, university professor. 
Table 5 Factors associated with adherence to the major dietary patterns according to ethnicity among two Iranian ethnic groups living in Urmia, Iran

\begin{tabular}{|c|c|c|c|c|c|c|c|c|c|c|c|c|}
\hline & \multicolumn{6}{|c|}{ Azeri Turks } & \multicolumn{6}{|c|}{ Kurd } \\
\hline & \multicolumn{2}{|c|}{ 'Traditional High SES' } & \multicolumn{2}{|c|}{ 'Transitional' } & \multicolumn{2}{|c|}{ 'Traditional Low SES' } & \multicolumn{2}{|c|}{ 'Traditional High SES' } & \multicolumn{2}{|c|}{ 'Transitional' } & \multicolumn{2}{|c|}{ 'Traditional Low SES' } \\
\hline & $B$ & $95 \% \mathrm{Cl}$ & $B$ & $95 \% \mathrm{Cl}$ & $B$ & $95 \% \mathrm{Cl}$ & $B$ & $95 \% \mathrm{Cl}$ & $B$ & $95 \% \mathrm{Cl}$ & $B$ & $95 \% \mathrm{Cl}$ \\
\hline $\begin{array}{l}\text { Age } \\
\text { Gender }\end{array}$ & $0 \cdot 21^{*}$ & $0.003,0.02$ & $-0.02^{\star \star}$ & $-0.03,-0.01$ & 0.004 & $-0.00-0.01$ & $0.01^{*}$ & $0.001,0.02^{*}$ & $-0.01^{\star \star}$ & $-0.02,-0.07$ & 0.002 & $-0.010,0.013$ \\
\hline $\begin{array}{l}\text { Gender } \\
\text { Female } \\
\text { Male }\end{array}$ & $\begin{array}{l}0.26 \\
0.00\end{array}$ & $\begin{array}{c}-0.10,0.51 \\
\text { Ref. }\end{array}$ & $\begin{array}{c}-0.006 \\
0.00\end{array}$ & $\begin{array}{c}-0.26,0.22 \\
\text { Ref. }\end{array}$ & $\begin{array}{l}-0.69^{\star \star \star} \\
0.00\end{array}$ & $\begin{array}{c}-0.96,-0.42 \\
\text { Ref. }\end{array}$ & $\begin{array}{l}0.44^{*} \\
0.00\end{array}$ & $\begin{array}{c}0.10,0.70 \\
\text { Ref. }\end{array}$ & $\begin{array}{c}-0.27^{*} \\
0.00\end{array}$ & $\begin{array}{c}-0.53,-0.02 \\
\text { Ref. }\end{array}$ & $\begin{array}{c}-0.39^{\star \star} \\
0.00\end{array}$ & $\begin{array}{c}-0.65,-0.13 \\
\text { Ref. }\end{array}$ \\
\hline Marital status & & & & & & & & & & & & \\
\hline $\begin{array}{l}\text { Married } \\
\text { Single/divorced/widow } \\
\text { Household size } \\
\text { Number of children } \\
\text { Duration of residence }\end{array}$ & $\begin{array}{c}0.08 \\
0.00 \\
-0.03 \\
0.03 \\
0.005\end{array}$ & $\begin{array}{c}-0.28,0.45 \\
\text { Ref. } \\
-0.13,0.06 \\
-0.07,0.13 \\
-0.002,0.11\end{array}$ & $\begin{array}{r}-0.14 \\
0.00 \\
-0.04 \\
0.01 \\
0.002\end{array}$ & $\begin{array}{c}-0.42,0.14 \\
\text { Ref. } \\
-0.11,0.03 \\
-0.06,0.09 \\
-0.002,0.007\end{array}$ & $\begin{array}{l}0.15 \\
0.00 \\
0.004 \\
-0.02 \\
-0.002\end{array}$ & $\begin{array}{l}-0.16,0.48 \\
\text { Ref. } \\
-0.08,0.09 \\
-0.11,0.06 \\
-0.00,0.09\end{array}$ & $\begin{array}{l}0.07 \\
0.00 \\
-0.01 \\
-0.05 \\
-0.003\end{array}$ & $\begin{array}{l}-0.29,0.43 \\
\text { Ref. } \\
-0.07,0.04 \\
-0.11,0.008 \\
-0.12,0.006\end{array}$ & $\begin{array}{l}-0.54^{\star \star *} \\
0.00 \\
-0.01 \\
-0.01 \\
-0.005\end{array}$ & $\begin{array}{l}-0.83,-0.2 \\
\text { Ref. } \\
-0.06,0.03 \\
-0.06,0.03 \\
-0.01,0.002\end{array}$ & $\begin{array}{l}0.40^{\star *} \\
0.00 \\
-0.03 \\
-0.01 \\
0.00\end{array}$ & $\begin{array}{c}0.11,0.69 \\
\text { Ref. } \\
-0.07,0.01 \\
-0.06,0.03 \\
-0.007,0.007\end{array}$ \\
\hline $\begin{array}{l}\text { Educational level } \\
\text { Illiterate and } \leq 5 \text { years } \\
6-12 \text { years } \\
\text { University degree }\end{array}$ & $\begin{array}{l}0.00 \\
0.30^{\star} \\
0.41^{\star *}\end{array}$ & $\begin{array}{c}\text { Ref. } \\
0.07,0.55 \\
0.11,0.71\end{array}$ & $\begin{array}{r}0.00 \\
0.04 \\
-0.01\end{array}$ & $\begin{array}{c}\text { Ref. } \\
-0.12,0.21 \\
-0.24,0.20\end{array}$ & $\begin{array}{c}0.00 \\
-0.20^{\star} \\
-0.29^{\star}\end{array}$ & $\begin{array}{c}\text { Ref. } \\
-0.40,-0.008 \\
-0.55,-0.04\end{array}$ & $\begin{array}{r}0.00 \\
0.09 \\
-0.09\end{array}$ & $\begin{array}{c}\text { Ref. } \\
-0.62,0.44 \\
-0.21,0.40\end{array}$ & $\begin{array}{l}0.00 \\
0.24^{\star *} \\
-0.11\end{array}$ & $\begin{array}{c}\text { Ref. } \\
0.000,0.48 \\
-0.52,0.29\end{array}$ & $\begin{array}{l}0.00 \\
-0.017 \\
-0.001\end{array}$ & $\begin{array}{c}\text { Ref. } \\
-0.26,0.23 \\
-0.40,0.40\end{array}$ \\
\hline $\begin{array}{l}\text { Job status } \\
\text { Unemployed, housewife } \\
\text { Job category } 1 \text { and } 2 \\
\text { Job category } 3 \text { and } 4 \\
\text { SES }\end{array}$ & $\begin{array}{r}0.00 \\
-0.13 \\
0.06\end{array}$ & $\begin{array}{c}\text { Ref. } \\
-0.32,0.44 \\
-045,0.17\end{array}$ & $\begin{array}{l}0.00 \\
0.44^{\star *} \\
0.27^{*}\end{array}$ & $\begin{array}{c}\text { Ref. } \\
0.15,0.73 \\
0.03,0.50\end{array}$ & $\begin{array}{r}0.00 \\
-0.20 \\
-0.22\end{array}$ & $\begin{array}{c}\text { Ref. } \\
-0.47,0.07 \\
-0.56,0.10\end{array}$ & $\begin{array}{r}0.00 \\
-0.16 \\
-0.38\end{array}$ & $\begin{array}{c}\text { Ref. } \\
-0.50,0.18 \\
-0.92,0.16\end{array}$ & $\begin{array}{l}0.00 \\
0.01 \\
0.23\end{array}$ & $\begin{array}{c}\text { Ref. } \\
-0.25,0.28 \\
-0.17,0.64\end{array}$ & $\begin{array}{r}0.00 \\
-0.28 \\
-0.08\end{array}$ & $\begin{array}{c}\text { Ref. } \\
-0.69,0 \cdot 13 \\
-0.35,0.18\end{array}$ \\
\hline $\begin{array}{l}\text { Low } \\
\text { Middle } \\
\text { High } \\
\text { Total income/capita (Rials) } \\
\text { Total expenses/capita (Rials) }\end{array}$ & $\begin{array}{l}0.00 \\
0.09 \\
0.12 \\
0.000 \\
0.000\end{array}$ & $\begin{array}{c}\text { Ref. } \\
-0 \cdot 16,0.35 \\
-0.14,0.39 \\
0.000,0.001 \\
-0.001,0.000\end{array}$ & $\begin{array}{r}0.00 \\
-0.18^{*} \\
-0.22^{*} \\
0.000 \\
0.000\end{array}$ & $\begin{array}{c}\text { Ref. } \\
-0.35,-0.008 \\
-0.43,-0.01 \\
0.000,0.001 \\
-0.001,0.000\end{array}$ & $\begin{array}{r}0.00 \\
0.04 \\
-0.03 \\
-1.21 \\
1.71\end{array}$ & $\begin{array}{c}\text { Ref. } \\
-0.18,0.27 \\
-0.26,0.18 \\
0.00,0.00 \\
0.00,0.00\end{array}$ & $\begin{array}{l}0.00 \\
0.09 \\
0.09 \\
0.001 \\
0.000\end{array}$ & $\begin{array}{c}\text { Ref. } \\
-0.11,0.35 \\
-0.20,-0.39 \\
0.000,0.001 \\
-0.001,0.001\end{array}$ & $\begin{array}{l}0.00 \\
0.01 \\
0.09 \\
2.10 \\
0.00\end{array}$ & $\begin{array}{c}\text { Ref. } \\
-0.19,0.22 \\
-0.14,0.33 \\
-0.001,0.001 \\
-0.001,0.001\end{array}$ & $\begin{array}{l}0.00 \\
0.10 \\
0.12 \\
-0.001^{\star *} \\
-7.69\end{array}$ & $\begin{array}{c}\text { Ref. } \\
-0.10,0.31 \\
-0.11,0.36 \\
-0.002,0.000 \\
-0.001,0.001\end{array}$ \\
\hline $\begin{array}{l}\text { Food insecurity status } \\
\text { Secure } \\
\text { Mild insecure } \\
\text { Moderate and severe }\end{array}$ & $\begin{array}{l}0.00 \\
-0.08 \\
-0.25^{\star}\end{array}$ & $\begin{array}{c}\text { Ref. } \\
-0.31,0.14 \\
-0.53,-0.02\end{array}$ & $\begin{array}{c}0.00 \\
-0.18^{*} \\
-0.22^{*}\end{array}$ & $\begin{array}{c}\text { Ref. } \\
-0.35,-0.008 \\
-0.04,-0.01\end{array}$ & $\begin{array}{l}0.00 \\
0.31^{\star \star} \\
0.39^{\star \star}\end{array}$ & $\begin{array}{c}\text { Ref. } \\
0.11,0.51 \\
0.14,0.63\end{array}$ & $\begin{array}{c}0.00 \\
-0.18 \\
-0.30^{\star}\end{array}$ & $\begin{array}{c}\text { Ref. } \\
-0.49,0.07 \\
-0.60,0.003\end{array}$ & $\begin{array}{l}0.00 \\
-0.32^{\star \star} \\
-0.26^{\star}\end{array}$ & $\begin{array}{c}\text { Ref. } \\
-0.53,-0.11 \\
-0.48,-0.04\end{array}$ & $\begin{array}{l}0.00 \\
0.24^{\star} \\
0.05\end{array}$ & $\begin{array}{c}\text { Ref. } \\
0.02,0.45 \\
-0.16,0.28\end{array}$ \\
\hline
\end{tabular}

SES, socio-economic status; $B$, regression coefficient (a positive coefficient implies a higher adherence to the pattern); Ref., reference category.

Data were adjusted for all variables shown in the table (demographic, socio-economic, lifestyle variables) and energy intake ( $\mathrm{kJ} / \mathrm{d}$ ).

Values were significantly different compared with the reference group of the listed variables: ${ }^{*} P<0.05,{ }^{\star \star} P<0.01,{ }^{* \star \star} P<0.001$. 
living in Urmia city. Two reflected traditional eating patterns; however, the third dietary pattern was a transitional pattern that represented evidence of acculturation towards Westernized dietary habits. These dietary patterns were associated with significant differences in SES and food insecurity status.

In the present study, the first extracted factor, the THS dietary pattern, explained the greatest percentage of variance. This pattern was named the 'Traditional High SES' due to the fact that food items loading highly on the factor included traditional healthy foods, including ash (usually containing green leafy vegetables and legumes) and dolma (prepared with vegetables) and traditional herbal beverages (pussy willow and lemon balm) that are abundantly cultivated in this region and are believed to have health benefits, but tend to be more expensive than other staple foods. Adherence to the THS dietary pattern was more common in Azeri participants compared with Kurds, but it was associated with SES among both groups. Azeri Turks are the main ethnic group in this city and have a longer duration of residence in Urmia, as well as higher SES relative to the Kurds living there. Previous reports have shown that the Kurds, who are immigrants from Kurdistan, the neighbouring province to West Azerbaijan, have lower social and economic status than Turks and a higher sense of social discrimination ${ }^{(15)}$. Interestingly, age was positively associated with the THS dietary pattern in Kurds, representing likely acculturation to Turk dietary habits. This dietary pattern has a healthy nature; it is relatively comparable to the 'healthy' or 'prudent' dietary pattern reported in previous studies on the Iranian population ${ }^{(30-34)}$ and in other countries ${ }^{(35-39)}$.

In contrast to the THS dietary pattern, another factor was based on traditional foods, but was labelled as 'Traditional Low SES'. The TLS dietary pattern contained foods that tend to be consumed more by lower-SES groups, including a high-fat traditional stew (abgoosht), all kinds of fats, refined grains, salt, as well as a few healthy food items such as whole grains and legumes. This dietary pattern consisted of energy-dense, low-cost foods and its constituent food groups are similar to those in the 'Iranian' dietary pattern previously described in Tehrani female teachers $^{(24)}$ and, to some extent, the 'Traditional' dietary pattern described in people from five metropolitan areas or central regions in $\operatorname{Iran}^{(30)}$ and in the Tehran Lipid and Glucose Study ${ }^{(22,40)}$. Although more Kurdish and fewer Azeri participants were in the top tertile of this dietary pattern, lower SES appeared to be the main determining factor influencing the tendency of people to consume this low-nutrient-quality dietary pattern in both ethnic groups. This dietary pattern was followed mainly by low-educated Azeri Turks in food-insecure households and Kurdish married men living in low-income families. In line with our findings, Tehrani teachers ${ }^{(24)}$ in the highest quintile of an 'Iranian' dietary pattern similar to our TLS dietary pattern had lower SES. Generally speaking, there is an inverse relationship between SES and energy density of consumed foods ${ }^{(41)}$; low-income people have restricted food choices, purchase poor-quality foods in terms of micronutrients ${ }^{(8)}$ and consume higher energy-dense food items high in fats and carbohydrates ${ }^{(42)}$.

The third dietary pattern was named 'Transitional'. This pattern was very similar to 'Western' dietary patterns introduced in previous studies in $\operatorname{Iran}^{(21,24,30,34,40)}$, as well as other Western and European nations ${ }^{(37,38,43-46)}$. This dietary pattern was highly consumed by higher-educated younger participants living in food-secure households in both ethnic groups. The distribution across tertiles of this dietary pattern did not differ by ethnicity. This pattern was highly loaded with high-protein food items, including red and organ meats, fast food, kebab, poultry, fish and shrimp. These are expensive food items in Iran; therefore, it is not surprising that this dietary pattern was consumed mainly by prosperous and food-secure households. Consistent with our findings, Tehrani teachers in the highest quintile of the 'Western' dietary pattern were more likely to have higher SES ${ }^{(24)}$. Also, the 'Western' dietary pattern among Haitians living in Canada was associated with higher $\mathrm{SES}^{(47)}$. Furthermore, among low-income adults living in Rio de Janeiro, lower household food insecurity and higher monthly per capita family income were positively associated with adherence to a 'Western' dietary pattern ${ }^{(41)}$.

Further, our findings are in accordance with several studies that have shown 'Western'-type dietary patterns to be followed more by younger individuals ${ }^{(30,46-49)}$. This may reflect acculturation to Western and/or urbanized eating habits and departure from traditional dietary habits ${ }^{(50)}$ to a more modern and convenient dietary pattern mainly by younger generations. As noted by Gilbert and Khokhar ${ }^{(8)}$, younger generations tend to change their eating habits by including fast foods, as they are seen to be convenient and reflective of independence. In contrast, older age has been shown to be associated with healthier traditional diets in many studies, as in our study, where it was associated with the THS dietary pattern ${ }^{(24,51,52)}$. Azeris who were employed, regardless of their job level and Kurds who were single ( $v$. married) were most likely to consume the 'Transitional' dietary pattern.

In the present study, gender was an important demographic factor related with adherence to a distinct dietary pattern among Kurds. Kurdish women were more likely to follow the THS while Kurdish men were more likely to follow the 'Transitional' or TLS dietary pattern. This suggests that Kurdish women pay more attention to healthy eating regardless of their SES and other demographic characteristics. Although, in Azeris, adherence to the THS and 'Transitional' dietary patterns did not differ significantly by gender, the TLS was mostly followed by men, as seen in Kurds. This suggests that Azeri women in lower-SES groups may have healthier eating habits as compared with men. These findings are in agreement with previous 
studies suggesting that women tend to adhere to healthier dietary patterns than men $38,39,43,49,53)$.

The present study has some limitations. First, the $80 \%$ response rate may pose response bias. One should take caution when generalizing the results to the studied population. Second, causality cannot be assigned to the associations observed herein due to the cross-sectional nature of the data. Explaining these relationships will require longitudinal studies that make the investigation of temporal sequences of events possible. Third, in assessing dietary intakes by FFQ some limitations, such as underreporting of usual intake or invalid reporting due to social desirability bias, should be considered ${ }^{(54)}$. Fourth, dietary pattern analysis involves several subjective or arbitrary decisions in categorizing food items into a certain food group, selecting the number of factors by various methods (eigenvalues, scree plots and interpretability) and finally in labelling of the extracted factors that may have an influence on the final content of the dietary patterns in the present study ${ }^{(4)}$.

\section{Conclusion}

Findings suggest that despite differences observed between the two studied ethnic groups in their tendency to consume a distinct dietary pattern (the THS dietary pattern in Azeri Turks and the TLS dietary pattern in Kurds), low SES and household food security level can affect the quality of their dietary patterns and this effect may be stronger than their cultural background. The 'Transitional' dietary pattern, however, appeared to be less affected by ethnicity and mostly influenced by the age of participants (younger generation). In general, findings emphasize the higher importance of social factors as compared with ethnic and biological factors in adhering to a high- or low-quality dietary pattern. In other words, understanding and interpreting determinants of dietary pattern(s) and food security in the multi-cultural context of Iran is dependent on considering socio-economic and political factors at micro and macro levels and their implications on ethnic groups' behaviours. The identified socio-economic barriers should be addressed in the design and administration of interventions to promote the adoption of healthier dietary choices in different ethnic groups.

\section{Acknowledgements}

Acknowledgements: The authors would like to thank the people who participated in this study. Also, they appreciate Dr Katherine Tucker for her special support in reviewing and English editing the manuscript. Financial support: This study was supported by National Nutrition and Food Technology Research Institute (NNFTRI) (grant number 501) for funding the study. The NNFTRI had no role in the design, analysis or writing of this article.
Conflict of interest: None of authors had a financial interest or professional affiliation that compromised the scientific integrity of this work. Authorship: N.O., A.R., M.G.-T. and R.M. formulated research questions and designed the study; A.R. collected data in the field; N.O. and A.R. analysed data and prepared the manuscript; H.E.-Z. and R.M. supervised statistical analysis; N.O., M.G.-T., R.M., S.G. and S.N.-S. supervised the study. This article resulted from the dissertation of A.R. as a PhD candidate in nutrition sciences. Ethics of human subject participation: This study was conducted according to the guidelines laid down in the Declaration of Helsinki and all procedures involving human subjects were approved by the Ethical Committees of the NNFTRI and the Faculty of Nutrition Sciences and Food Technology, Shahid Beheshti University of Medical Sciences. Written informed consent was obtained from all participants.

\section{References}

1. Panagiotakos DB, Chrysohoou C, Pitsavos C et al. (2006) Association between the prevalence of obesity and adherence to the Mediterranean diet: the ATTICA study. Nutrition 22, 449-456.

2. Schols AM (1997) Nutrition and outcome in chronic respiratory disease. Nutrition 13, 161-163.

3. World Health Organization (1998) Preparation and Use of Food-Based Dietary Guidelines. Joint FAO/WHO Consultation, WHO Technical Report Series no. 880. Geneva: WHO.

4. Hu FB (2002) Dietary pattern analysis: a new direction in nutritional epidemiology. Curr Opin Lipidol 13, 3-9.

5. Hu FB, Rimm EB, Stampfer MJ et al. (2000) Prospective study of major dietary patterns and risk of coronary heart disease in men. Am J Clin Nutr 72, 912-921.

6. Nicklas T, Webber L, Thompson B et al. (1989) A multivariate model for assessing eating patterns and their relationship to cardiovascular risk factors: the Bogalusa Heart Study. Am J Clin Nutr 49, 1320-1327.

7. Yang EJ, Kerver JM \& Song WO (2005) Dietary patterns of Korean Americans described by factor analysis. $\mathrm{J}$ Am Coll Nutr 24, 115-121.

8. Gilbert PA \& Khokhar S (2008) Changing dietary habits of ethnic groups in Europe and implications for health. Nutr Rev 66, 203-215.

9. Rezazadeh A, Rashidkhani B \& Omidvar N (2010) Association of major dietary patterns with socioeconomic and lifestyle factors of adult women living in Tehran, Iran. Nutrition 26, 337-341.

10. Huijbregts P, Feskens E, Räsänen L et al. (1997) Dietary pattern and 20 year mortality in elderly men in Finland, Italy, and The Netherlands: longitudinal cohort study. BMJ 315, 13-17.

11. Marmot MG, Stansfeld S, Patel C et al. (1991) Health inequalities among British civil servants: the Whitehall II study. Lancet 337, 1387-1393.

12. Margetts B, Thompson R, Speller V et al. (1998) Factors which influence 'healthy' eating patterns: results from the 1993 Health Education Authority health and lifestyle survey in England. Public Health Nutr 1, 193-198.

13. Azizi F, Azadbakht L \& Mirmiran P (2005) Trends in overweight, obesity and central fat accumulation among Tehranian adults between 1998-1999 and 2001-2002: Tehran Lipid and Glucose Study. Ann Nutr Metab 49, 3-8. 
14. Iran Council of Public Culture (2012) Plan of Examine and Assessment of the General Culture of the Country Indices (not registerd indices), West Azerbaijan Province. Tehran: Iran Council of Public Culture.

15. Hosseini $\mathrm{H}$ (2008) Ethnicity and fertility: explaining the behavior of fertility in Turk and Kurd females leaving in the city of Urmia. PhD Thesis, Tehran University.

16. Ministry of Health and Medical Education (2008) Report on Non-Communicable Disease Risk Factors in 30 Provinces in 2006. Tehran: Ministry of Health and Medical Education.

17. Mohammadi F (2012) Measurement and modeling of household food security in urban households in the city of Tehran. PhD Thesis, Shahid Beheshti University of Medical Sciences and Health Services.

18. Goldberg G, Black A, Jebb S et al. (1991) Critical evaluation of energy intake data using fundamental principles of energy physiology: 1 . Derivation of cut-off limits to identify under-recording. Eur J Clin Nutr 45, 569-581.

19. Willett WC, Sampson L, Stampfer MJ et al. (1985) Reproducibility and validity of a semiquantitative food frequency questionnaire. Am J Epidemiol 122, 51-65.

20. Esfahani FH, Asghari G, Mirmiran P et al. (2009) Reproducibility and relative validity of food group intake in a food frequency questionnaire developed for the Tehran Lipid and Glucose Study. J Epidemiol 20, 150-158.

21. Mirmiran P, Hosseini Esfahani F, Mehrabi Y et al. (2010) Reliability and relative validity of an FFQ for nutrients in the Tehran Lipid and Glucose Study. Public Health Nutr 13, 654-662.

22. Asghari G, Rezazadeh A, Hosseini-Esfahani F et al. (2012) Reliability, comparative validity and stability of dietary patterns derived from an FFQ in the Tehran Lipid and Glucose Study. Br J Nutr 108, 1109-1117.

23. Lopez-Garcia E, Schulze MB, Fung TT et al. (2004) Major dietary patterns are related to plasma concentrations of markers of inflammation and endothelial dysfunction. $A m \mathrm{~J}$ Clin Nutr 80, 1029-1035.

24. Esmaillzadeh A \& Azadbakht L (2008) Major dietary patterns in relation to general obesity and central adiposity among Iranian women. J Nutr 138, 358-363.

25. Rezazadeh A \& Rashidkhani B (2010) The association of general and central obesity with major dietary patterns in adult women living in Tehran, Iran. J Nutr Sci Vitaminol (Tokyo) 56, 132-138.

26. Eini-Zainab H (2013) Evaluation of Multivariate Indices and the Total Fertility Rate in Iran. Tehran: Asia-Pacific Population Research Center.

27. Wunderlich GS \& Norwood JL (2006) Food Insecurity and Hunger in the United States: An Assessment of the Measure. Washington, DC: National Academies Press.

28. Mohammadi F, Omidvar N, Houshiar-Rad A et al. (2011) Validity of an adapted Household Food Insecurity Access Scale in urban households in Iran. Public Health Nutr 15, 149-157.

29. Kim J-O \& Mueller CW (1978) Factor Analysis: Statistical Methods and Practical Issues. Sage University Paper Series on Quantitative Applications on the Social Sciences no. 07-014. Beverly Hills, CA: Sage.

30. Jafarabadi M, Ghazizahedi S, Mohajeri S et al. (2014) Comparison of dietary pattern in different provinces of Iran. Nationalpark-Forschung in der Schweiz (Switz Res Park J) 102, 1823-1838.

31. Aghajani-Delavar M, Lye M-S, Khor GL et al. (2009) Dietary patterns and the metabolic syndrome in middle aged women, Babol, Iran. Asia Pac J Clin Nutr 18 , 285-292.

32. Rezazadeh A (2014) Designing and testing of a multilevel model to explain the effects of neighborhood, household and individual on anthropometric factors in men and women living in the city of Urmia. PhD Thesis, Shahid
Beheshti University of Medical Sciences and Health Services.

33. Haidari F, Shirbeigi E, Cheraghpour M et al. (2014) Association of dietary patterns with body mass index, waist circumference, and blood pressure in an adult population in Ahvaz, Iran. Saudi Med J 35, 967-974.

34. Mohammadifard N, Sarrafzadegan N, Nouri F et al. (2012) Using factor analysis to identify dietary patterns in Iranian adults: Isfahan Healthy Heart Program. Int J Public Health 57, 235-241.

35. Arruda SP, da Silva AA, Kac G et al. (2014) Socioeconomic and demographic factors are associated with dietary patterns in a cohort of young Brazilian adults. BMC Public Health 14, 654 .

36. Fonseca MJ, Gaio R, Lopes C et al. (2012) Association between dietary patterns and metabolic syndrome in a sample of Portuguese adults. Nutr J 11, 64.

37. Richter A, Heidemann C, Schulze MB et al. (2012) Dietary patterns of adolescents in Germany - associations with nutrient intake and other health related lifestyle characteristics. BMC Pediatr 12, 35.

38. Brenner DR, Boucher BA, Kreiger N et al. (2011) Dietary patterns in an ethnoculturally diverse population of young Canadian adults. Can J Diet Pract Res 72, e161-e168.

39. Naja F, Nasreddine L, Itani L et al. (2011) Dietary patterns and their association with obesity and sociodemographic factors in a national sample of Lebanese adults. Public Health Nutr 14, 1570-1578.

40. Mirmiran P, Hosseini-Esfahani F, Jazayeri A et al. (2008) Changing dietary patterns and their relation to changes in weight and body mass index in adults living in District 13 of Tehran. Iran J Nutr Sci Food Technol 2, 67-80.

41. Cunha DB, Sichieri R, de Almeida RMVR et al. (2011) Factors associated with dietary patterns among lowincome adults. Public Health Nutr 14, 1579-1585.

42. Drewnowski A \& Specter S (2004) Poverty and obesity: the role of energy density and energy costs. Am J Clin Nutr $\mathbf{7 9}$, 6-16.

43. Gimeno SGA, Andreoni S, Ferreira SRG et al. (2010) Assessing food dietary intakes in Japanese-Brazilians using factor analysis. Cad Saude Publica 26, 2157-2167.

44. Hu FB, Rimm E, Smith-Warner SA et al. (1999) Reproducibility and validity of dietary patterns assessed with a foodfrequency questionnaire. Am J Clin Nutr 69, 243-249.

45. Khani BR, Ye W, Terry P et al. (2004) Reproducibility and validity of major dietary patterns among Swedish women assessed with a food-frequency questionnaire. J Nutr $\mathbf{1 3 4}$, $1541-1545$.

46. Sánchez-Villegas A, Delgado-Rodriguez M, MartínezGonzález MÁ et al. (2003) Gender, age, socio-demographic and lifestyle factors associated with major dietary patterns in the Spanish Project SUN (Seguimiento Universidad de Navarra). Eur J Clin Nutr 57, 285-292.

47. Desilets M-C, Rivard M, Shatenstein B et al. (2007) Dietary transition stages based on eating patterns and diet quality among Haitians of Montreal, Canada. Public Health Nutr 10, 454-463.

48. Park S-Y, Murphy SP, Wilkens LR et al. (2005) Dietary patterns using the Food Guide Pyramid groups are associated with sociodemographic and lifestyle factors: the multiethnic cohort study. J Nutr 135, 843-849.

49. Northstone K, Smith AD, Cribb VL et al. (2014) Dietary patterns in UK adolescents obtained from a dual-source FFQ and their associations with socio-economic position, nutrient intake and modes of eating. Public Health Nutr 17, 1476-1485.

50. Ghassemi H, Harrison G \& Mohammad K (2002) An accelerated nutrition transition in Iran. Public Health Nutr $\mathbf{5}$, 149-155. 
51. Vengiau G, Umezaki M, Phuanukoonnon S et al. (2014) Associations of socioeconomic status with diet and physical activity in migrant Bougainvilleans in Port Moresby, Papua New Guinea. Ecol Food Nutr 53, 471-483.

52. Abu-Saad K, Murad H, Lubin F et al. (2012) Jews and Arabs in the same region in Israel exhibit major differences in dietary patterns. J Nutr 142, 2175-2181.
53. Newby P \& Tucker KL (2004) Empirically derived eating patterns using factor or cluster analysis: a review. Nutr Rev 62, 177-203.

54. Kerver JM, Yang EJ, Bianchi L et al. (2003) Dietary patterns associated with risk factors for cardiovascular disease in healthy US adults. Am J Clin Nutr 78, $1103-1110$. 